\title{
Fast level-set based image segmentation using coherent propagation
}

\author{
Chunliang Wang, Hans Frimmel and Örjan Smedby
}

\section{Linköping University Post Print}

\section{Tweet}

N.B.: When citing this work, cite the original article.

Original Publication:

Chunliang Wang, Hans Frimmel and Örjan Smedby, Fast level-set based image segmentation using coherent propagation, 2014, Medical physics (Lancaster), (41), 7, 073501.

http://dx.doi.org/10.1118/1.4881315

Copyright: American Association of Physicists in Medicine: Medical Physics http://www.aapm.org/main.asp

Postprint available at: Linköping University Electronic Press

http://urn.kb.se/resolve?urn=urn:nbn:se:liu:diva-109599 


\title{
Fast Level-set Based Image Segmentation Using Coherent Propagation
}

\author{
Chunliang Wang ${ }^{1,2}$, Hans Frimmel ${ }^{3}$ and Örjan Smedby ${ }^{1,2}$ \\ ${ }^{1}$ Center for Medical Image Science and Visualization (CMIV), Linköping University, Sweden \\ ${ }^{2}$ Division of Radiological Sciences (IMH), Linköping University, Sweden \\ ${ }^{3}$ Division of Scientific Computing, Uppsala University, Sweden
}

Purpose: The level-set method is known to require long computation time for 3D image segmentation, which limits its usage in clinical workflow. The goal of this study was to develop a fast level set algorithm based on the coherent propagation method and explore its character using clinical datasets. Methods: The coherent propagation algorithm allows level set functions to converge faster by forcing the contour to move monotonically according to a predicted developing trend. Repeated temporary backwards propagation, caused by noise or numerical errors, is then avoided. It also makes it possible to detect local convergence, so that the parts of the boundary that have reached their final position can be excluded in subsequent iterations, thus reducing computation time. To compensate for the overshoot error, forward and backward coherent propagation is repeated periodically. This can result in fluctuations of great magnitude in parts of the contour. In this paper, a new gradual convergence scheme using a damping factor is proposed to address this problem. The new algorithm is also generalized to non-narrow band cases. Finally, the coherent propagation approach is combined with a new distance-regularized level set, which eliminates the needs of re-initialization of the distance. Results: Compared with the sparse field method implemented in the widely available ITKSnap software, the proposed algorithm is about 10 times faster when used for brain segmentation and about 100 times faster for aorta segmentation. Using a multi-resolution approach, the new method achieved 50 times speed-up in liver segmentation. The Dice coefficient between the proposed method and the sparse field method is above $99 \%$ in most cases. Conclusion: A generalized coherent propagation algorithm for level set evolution yielded substantial improvement in processing time with both synthetic datasets and medical images.

Keywords: Image segmentation, level set, coherent propagation, monotonic propagation, medical imaging. 


\section{Introduction}

As a member of the active contour family, the level set method has been widely used as a promising segmentation tool in various image processing tasks, such as medical image analysis, motion tracking and 3D modeling. ${ }^{1-4}$ Unlike other types of active contours or snakes, level sets use an implicit function, given by the distance to the zero level set, to represent the boundary of an object in an image. This enables the level set method to handle automatically complicated topological changes of the target object, and increases the accuracy, as the sample step length of the contour is no greater than one pixel/voxel. However, it also results in much longer computation times than other, explicit, active contour methods, and therefore its usefulness in time-critical tasks, such as clinical image analysis and real-time motion tracking, is limited. To accelerate its performance, various strategies have been reported in the literature. These methods can be roughly divided into three categories.

40 The first category of methods tries to speed up the level set method by skipping the unnecessary computations in certain points. The narrow-band level set method ${ }^{5}$ is one such method, in which the computation of the level-set function is limited to a band a few pixels wide instead of the whole image. It was later extended to the sparse-field level set, ${ }^{6}$ where the narrow band is only one pixel wide and the level set function is reinitialized with a distance transform in each iteration. The computation time can be reduced even further by only updating the level function around the point with minimum arrival time as suggested by Deng et al., ${ }^{7}$ (793 s to segment two rings in a $100^{3}$ volume) or by skipping the calculation in certain converged parts of the narrow band, as reported by $\mathrm{Vu}$ et al. ${ }^{8}$ (39 47 $\mathrm{s}$ to segment skull in $512^{2} \mathrm{CT}$ slices) and Roberts et al. ${ }^{9}$ (7 $\mathrm{s}$ to segment brain in a $256^{3}$ volume on GPU).

50 The second category of methods attempt to speed up the convergence of the discrete integration by using faster partial differential equation (PDE) solvers or solving the PDE in a discrete manner. For example, additive operator splitting-(AOS-)based methods use an implicit scheme to achieve 
intrinsic stability and therefore allow a larger step length during the gradient descent ${ }^{10}$ ( 3 min to segment brain in a $192 \times 250 \times 170$ volume). Gibou et al. ${ }^{11}$ proposed a hybrid k-means level set

55 which de-couples the image term from the regularization term (curvature term), and propagates the region with no curvature regularization for some time and then performs the time-consuming step of mean curvature motion for fewer iterations $(0.3 \mathrm{~s}$ to segment left hemi-sphere in a $192 \times 128 \times 61$ volume). Shi et al. ${ }^{12,13}$ developed a similar approach but with more approximation ( $4.4 \mathrm{~s}$ to segment white matter in a $181 \times 217 \times 181$ volume). They also circumvented solving the PDE by using

60 diffusion or a discrete scheme. ${ }^{12}$ A similar strategy is also used by Bernard et al. ${ }^{14}$ in their variational B-spline level-set method (540 s to segment calcaneus bone in 3D micro CT). Various approximations of the speed function and mean curvature calculation were also proposed by different groups. ${ }^{15-17}$ However, such approximations will often sacrifice accuracy to gain speed.

The third category of methods tries to avoid re-initialization of the level set function by 65 incorporating the signed distance constraint into the PDE as an additional regularization term. As pointed out by Li et al., ${ }^{18}$ this not only eliminates the needs of periodic re-initialization, but also allows the level set to evolve in a larger step length and results in faster convergence $(0.23 \mathrm{~s}$ to segment bladder from a $107 \times 180$ MRI slice). These strategies, or combinations of several strategies, were reported to reduce the computation by one or two orders of magnitude.

70 Besides optimizations done on the algorithm level, the recent development in parallel computing, in particular GPU computing, has introduced a new way to improve the segmentation speed. Significant speed-up was reported in a number of studies on implementing level-set on GPU.9, 19, 20 However, in our experience, the performance of most optimized algorithms on 3D medical data is still not adequate for clinical practice. For example, typical $3 \mathrm{D}$ brain segmentation $\left(256^{3}\right)$ takes 75 more than 5 minutes on an ordinary PC and about 2 minutes on a GPU, when using the sparse field approach. $^{19}$ 
In the study of Wang et al., ${ }^{21}$ a new fast sparse-field level set algorithm with coherent propagation was proposed, which limits the developing trend of a level set function to be consistent on each pixel/voxel throughout a whole propagation period, i.e. the level set function can only be 80 either increasing or decreasing (corresponding to a locally shrinking or expanding contour) in one point. This coherent propagation is then repeated in opposite directions periodically. A permanent value is assigned to a point by calculating the mean value of the maximum and minimum during the last period. This strategy stops the contour's wiggling behavior, which often occurs when certain points during the propagation move faster than the neighbors, as the curvature force will move them

85 backwards even though the whole neighborhood will eventually move forwards. Faster convergence was then achieved. ${ }^{21}$ Coherent propagation also makes it possible to detect local convergence within each period, since when the contour reaches the object border, the propagation speed will change sign, turning opposite to the predicted direction. These points can then be excluded from the computation in the remaining iterations. Thanks to these two strategies, significant speedup (over

9010 times) was achieved for 3D vessel segmentation compared to the conventional sparse field level set method implemented in the widely available ITKSnap software. ${ }^{1}$

In this report, we explore further the character of the coherent propagation method and generalize it for non-narrow-band implementations by introducing a new gradual convergence scheme. Compared with the original algorihtm, ${ }^{21}$ the new convergence scheme simplifies the 95 implementation considerably and enables more general applications that cannot be solved with narrow band approaches. The new algorithm can also be combined with the distance-regularized level set function ${ }^{18}$ to achieve even better performance. In section 2.1 , we first quickly review the coherent propagation method proposed in the study of Wang et al. ${ }^{21}$ In section 2.2 , the issue of fluctuation during the periodic bi-directional propagation, which often occurs in those regions of an 100 image where the object's boundary is blurred, is addressed. A damping factor was introduced during the periodic coherent propagation to stop this irregularity. In section 2.3 and 2.4, a new 
generalized coherent propagation algorithm for dense field level set evolution was proposed and combined with a distance-regularized level set function to benefit those cases where narrow-band approaches are not applicable. In section 3, we demonstrate the performance character and accuracy of the proposed algorithm using both synthetic and clinical datasets. Substantial speed-up was observed in our preliminary tests, while the segmentation accuracy was not sacrificed. The new algorithm will hopefully extend the scope of level-set techniques to more time-critical applications.

\section{Method}

\subsection{Level set with coherent propagation}

110 Active contours can be seen as a rubber band (in 2D) or an elastic balloon (in 3D), and the image segmentation using active contours is similar to the rubber band being stretched towards the object boundary by an external force related to image information (e.g. intensity), while the internal elastic force tries to keep the contour smooth. ${ }^{22}$ The object's contour can be represented explicitly as 2D curves or 3D meshes. In the level-set method, however, it is represented by a dynamic implicit

115 function (level set function), which is usually a signed distance function. For the convenience of discussion, in this paper we define the level set function as negative inside and positive outside the contour. By iteratively evolving this function according to external and internal forces over artificial time, the 0 -level set can be deformed to the shape of the object of interest. This evolution can be expressed as a PDE (Eq. 1).

$$
\frac{\partial \phi}{\partial t}=v_{t}
$$

120 Both the internal and external forces are incorporated in the speed function and balanced using weighting factors. Typical examples are the region-based level set function (Eq. 2) $)^{19,23}$ and gradient-based level set function (Eq. 3) ${ }^{24}$ : 


$$
\begin{gathered}
v_{t}=\alpha \kappa|\nabla \phi|+(\varepsilon-|I-T|)|\nabla \phi| \\
v_{t}=\mathrm{g}(c+\kappa)|\nabla \phi|+\nabla \mathrm{g} \cdot \nabla \phi
\end{gathered}
$$

In Eq. 2, $\alpha$ is a weighting factor, $\kappa$ is the mean curvature, $I$ is the intensity of the input image, $T$ is the center of the threshold window and $\varepsilon$ is the width of the threshold window. Eq. 3 is known as 125 geodesic active contour, where $g$ the edge indicator function which is strictly decreasing such that when $g \rightarrow 0$ as $|\nabla I| \rightarrow \infty, c$ is a balloon force and $\kappa$ is the mean curvature. These PDEs are usually solved using the gradient descent approach, i.e.

$$
\phi_{t+\Delta t}=\phi+v_{t} \Delta t
$$

While $\Delta t$ is usually set to be the same across the image, $v_{t}$ is different for each pixel/voxel during the evolution. When a relatively large $\Delta t$ is used, the propagation front will assume a zigzag shape.

130 This is almost inevitable, due to noise and numerical errors. The faster points in the neighborhood (point 2 and 4 in Fig. 1(a)) will be "pulled back" by the curvature force in the next iteration, even if the trend of the local segment is to move forward when the neighbor points catch up. This temporary backwards propagation significantly slows down the propagation.

In the study of Wang et al., ${ }^{11}$ a monotonic speed function was proposed:

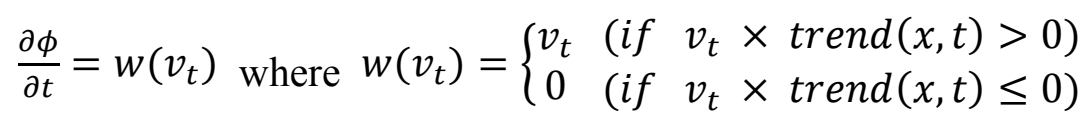

Here, "trend" represents whether the local contour is expanding or shrinking. Its value can only be 1 or -1 ; as in level set the function $v_{t}$ is a scalar. For the sparse-field/narrow-band level set, "trend" for each pixel/voxel is initialized when it is added for the first time to the narrow band, using $\operatorname{sign}\left(v_{t}\right)$, and passed on to the neighbors during propagation. Its values need not be the same across the image; however, once set, it cannot be changed, which prevents the contour from passing a point twice. 
With this monotonic speed function, the faster points (point 2 and 4 in Fig. 1(b)) will not move back by the curvature force (since $v_{t} \times$ trend $<0$ ), but stay in place and wait for the neighbors to catch up. Then, as the contour becomes flat again, $v_{t}$ at point 2 and 4 will have the same sign as trend, and all points can move forward together.

145 In this way, the expanding parts of the contour keep expanding, and the shrinking parts keep shrinking in a coherent manner, until they reach the boundary of the segmented object. As the force related to extrinsic factors will change sign when the contour has crossed the boundary, these parts of the contour can no longer expand or shrink. At the same time, the curvature force will prevent the contour from growing into corners by changing their sign and never changing back. The propagation stops naturally when $w\left(v_{t}\right)$ becomes 0 everywhere on the contour.

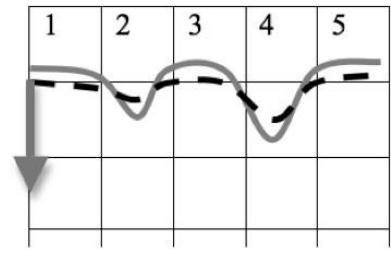

(a)

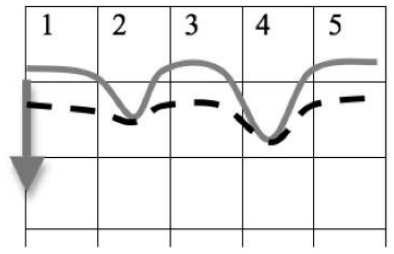

(b)

Figure 1. (a) Example of temporary backwards propagation. (b) Coherent propagation. The arrow indicates the global propagation trend, the gray line is the current contour, and the dashed line is the contour after one iteration.

Coherent propagation also makes it possible to detect local convergence, since if the contour reaches the border, $w\left(v_{t}\right)$ of that part of contour will become 0 . If no neighbor changes it value, $w\left(v_{t}\right)$ will always return 0 in the subsequent iterations. These points with zero speed can simply be "put to sleep" and excluded from further updating. As these points may be just in a temporal "equilibrium" $\left(w\left(v_{t}\right)=0\right)$, a wake-up mechanism is implemented to allow all changed points $\left(w\left(v_{t}\right) \neq 0\right)$ to wake up sleeping points in a 1-2 pixel/voxel wide neighborhood.

\subsection{Overshoot and gradual convergence with a damping factor}

In a conventional level set algorithm, when the zero level set crosses the boundary of an object, the external force will try to move the contour back. With coherent propagation, on the other hand, 
the speed of these points is set to zero and they are "put to sleep". This clearly results in a small

165 overshoot error for most parts of the contour. A periodic forward and backward propagation is then needed to compensate for these errors. A new period starts when $w\left(v_{t}\right)$ is 0 (i.e. $v_{t} \times$ trend $\leq 0$ ) for every point on the narrow band. The "trend" of the points on the narrow band will change sign at the same time. When the zero level set is located on a sharp edge, the propagation will stop soon, which means that $v_{t}$ changes from positive to negative (or vice versa). A permanent value is assigned to points by calculating the mean value of the maximum and the minimum during the last period. In some areas where the curvature force is greater than the external force, e.g. in sharp corners or the intensity close to the threshold ( $\varepsilon-|I-T|$ close to zero), the contour can jump in larger steps (Fig. 2 B). In the study of Wang et al., ${ }^{21}$ this so-called elastic region was solved by using a forced convergence step, where the jumping was gradually stopped from the region moving in small steps (1 pixel/voxel) for several periods. The unstopped region will jump in smaller steps when the neighbor has been assigned to a permanent value. However, the cause of the fluctuation was not well investigated. Further close analysis reveals that this type of fluctuation is caused by a small overshoot in each iteration and the fact that the points are only allowed to propagate in one direction. As illustrated in Fig. 2 A, the points in concave regions (a, c, e) can move too far in just one iteration, which will cause the convex regions $(b, d)$ to become concave. In the conventional level set method, this does not matter: as two groups of points are moving in opposite directions at the same time, that results in a dynamic equilibrium. With coherent propagation, points are only allowed to propagate in one direction, which prevents overshooting points (a, c, e) to come back. In the worst scenario, points $b$ and $d$ will in the next iteration be moved even further to minimize the arc length (curvature force). This error can be magnified if the elastic region is large. Although the force convergence method will still work (Fig. 2 C), it may take many periods to gradually stop this jumping, especially in cases where the boundary between objects is indistinct. One way to avoid the overshoot problem is to reduce the step length, i.e. $\Delta t \times v_{t}$, in each iteration (Fig. 2 D). However, it 
has to be small enough to totally avoid the fluctuation. In our experience, most elastic regions become stationary when $\Delta t \times v_{t}$ is always less than 0.1 pixel/voxel. Evidently, this will increase the number of iterations needed for the contour to reach the border $(0.5-1.0$ is normally chosen for the limit of the step length). Therefore, we proposed to reduce the maximum step length $s_{\max }$ gradually from period to period. In the first period, the maximum step length is set relatively high, e.g. 0.8 (Note that $\Delta t \times v_{t}$ must be less than 1.0 to maintain the stability of the level set). ${ }^{22}$ It is then scaled by a damping factor $d(d<1.0)$ in the end of each period, which causes $s_{\max }$ to decrease exponentially over periods. This will make the contour grow at high speed in the first period, and the overshooting problem is reduced gradually. After a few periods (to our knowledge 4-6, when $s_{\max }<0.1$ ), the periodic coherent propagation can be stopped, and the final level set value for each point is calculated as the mean value of the initial and final value in the last period.

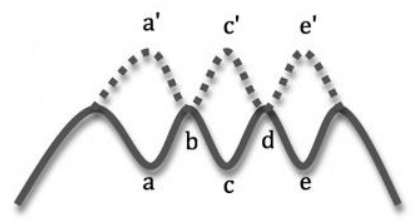

(a)

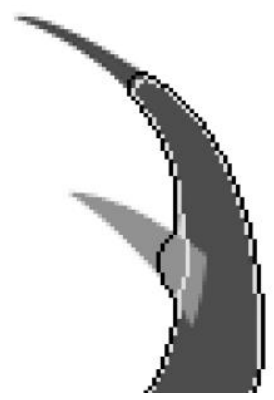

(b)

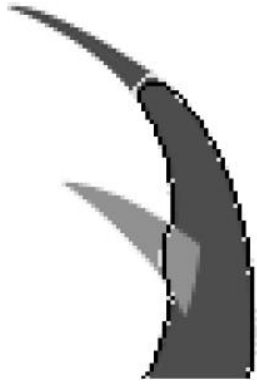

(c)

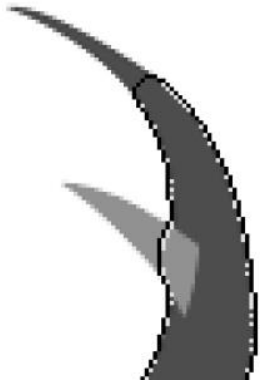

(d)

Figure 2. (a) An example of the overshoot problem, solid line represents the current zero level set, dash line represents the level set after the next iteration. (b) Narrow bands at the end of forward (dark line) and backward (bright gray line) period with $s_{\max }=1.0$. (c) Narrow band from force converged algorithm (dark line, $s_{\max }=1.0$ ) compared with that from the conventional sparse field (bright gray line, $s_{\max }=0.5$ ). (d) Narrow band from single period coherent propagation with small step length (dark line, $s_{\max }=0.1$ ) compared with that from the conventional sparse field (bright gray line, $s_{\max }=0.5$ ).

\subsection{Generalized coherent propagation}

It can easily be shown that Eq. 5 is applicable to any level of a level set function, not only to the zero level set. Using the damping factor instead of the wiggling counter eliminates the need to keep tracking the zero level set. Therefore, the coherent propagation can be extended to a more general PDE solver. In this section we propose a generalized algorithm based on the coherent propagation 
method. Given an input image $I=(C, f)$, where $C$ is a collection of image point coordinates and $f$ is the corresponding intensity function to the points, and a pre-define formula for the speed function $215 v_{t}$, the proposed algorithm is summarized in Table I.

Table I Pseudo-code for the main loop of coherent propagation
1. initialize level set function $\phi$
2. $s_{\max }=$ initial step length $s_{1}$
3. do
4. $\quad \phi_{\text {previous }}=\phi$
5. estimate $\operatorname{trend}(x)$ for all $x \in C$
6. coherent_propagate $\left(\phi, \text { trend }, s_{\max }\right)^{*}$
7. $s_{\max }=s_{\max } \times d$
8. while $\left(s_{\max }<\right.$ finest step length $\left.s_{2}\right)$
9. $\phi=\left(\phi+\phi_{\text {previous }}\right) / 2$

\section{*: given in Table II}

The main loop of the new algorithm consists of two major steps, estimating the trend map for every pixel/voxel and running the coherent propagation. The maximum step length $s_{\max }$ is also gradually decreased from $s_{1}$ to $s_{2}$ using a damping factor $d\left(0<s_{2}<s_{1}<1.0\right.$ and $\left.0<d<1.0\right)$. The trend map of the level set's movement can be predicted based on some heuristic criteria. Here we propose to use the sign of the mean momentum in the neighborhood, as suggested in Eq. 6, where the function $N(x, r)$ returns all the points within distance $r$ to $x$. Note that if $r$ is set to big enough, the sign of trends of all points will be unified, which means the level set function will be elevated or 225 depressed on all points simultaneously. In such cases, re-estimation in the subsequent periods can simply be replaced by switching the sign of trend, while the initial value can alternatively be set manually by the user. Another extreme is to set $r$ to 1 , which is the implementation used in the previous report. ${ }^{21}$ As pointed out by Wang et al., ${ }^{21}$ a single point with opposite trend will not stop 
the propagation of the level sets, due to the level set's ability to change topology. However, in an extremely noisy scenario and combined with a higher curvature force factor, smaller $r$ could give an erroneous estimation and slow down the propagation in the first few periods.

$$
\operatorname{trend}(x)=\operatorname{sign}\left(\sum_{s \in N\left(x, r_{1}\right)} v(s)\right)
$$

Inside the function coherent_propagate $\left(\phi\right.$, trend, $\left.s_{\max }\right)$, only the points moving towards the predicted direction get updated, otherwise they are set to "sleep". The pseudo-code is given in Table II. As $v(x)$ may change signs when the neighbors change, the points that are neighboring (within a distance of $r_{2}$ ) to a changing point are waked up. The iterative operation stops when the level set function converges or the iteration number goes beyond a threshold $n . n$ can also vary from period to period like $s_{\max }$ since fewer iterations are needed to reach the boundary after the first period in which the zero level set travels the greatest distance. The limit on iteration is not necessary in the case where the level set function does converge, such as in most sparse field cases and the distance regularized dense field level set. ${ }^{18}$ However, in most other types of dense field level set function, where there is no regularization of the level set, the inside part of the level can keep decreasing and the outside part keep increasing, so that no convergence is obtained. To some extent, $n$ is similar to the limitation on the number of iterations in the conventional level set algorithms. Note that if we set $n=1$ and $d=1$, then the algorithm degenerates to the conventional gradient descent algorithm.

1. $\operatorname{set} \operatorname{status}(x)=$ active for all $x \in C$

2. iteration $=0$

3. do

4. changed_list.clear()

5. for all $x \in C$ and $\operatorname{status}(x)=\operatorname{active}$ 


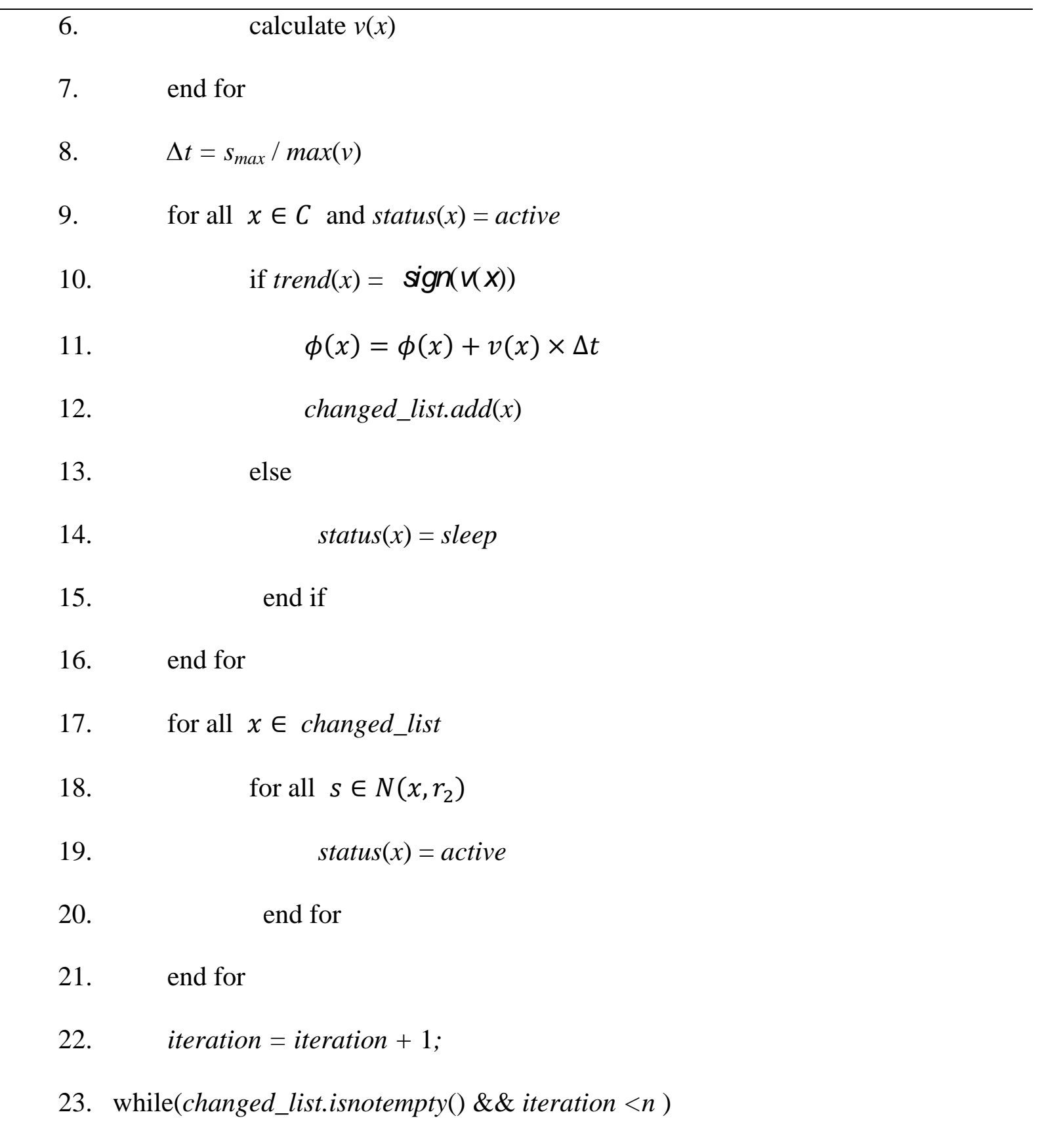

\subsection{Distance regularized level set without re-initialization}

In the dense field case, the PDE needs to converge for the coherent propagation to work better. However, the conventional region- or gradient-based level set evolution will normally not converge, since there is no regularization term for the level set function itself. After a few iterations, the level 250 set function will shift from the distance function. This can be solved by re-initialization; however, such an operation makes it difficult to detect local convergence except on the zero level set, using 
the proposed coherent propagation method. Although a fixed number of iterations can be used to terminate a period, such a parameter is case-dependent as in the conventional level set algorithms. Inspired by Li's et al. work, we propose a new distance regularized level set function to solve the problem. Similar to the method proposed by Li et al., ${ }^{18}$ an additional energy function is added to regularize the level set function (Eq. 7). Unlike Li's method, ${ }^{18}$ the additional term $d_{r}$ is not introduced to regularize $|\nabla \phi|$ to 1 or 0 , but to limit the level set function $\phi$ between the "floor" ($\tau)$ and the "ceiling" $\tau$ (Eq. 9). An additional condition is added so that, when $\phi$ is between $-\tau$ and $\tau$ and $|\nabla \phi|<\rho_{s}$, it will also be attracted either to the "floor" or to the "ceiling". This will generate a 260 platform-shaped $\phi$ eventually similar to Li's method (Fig. 3), even though the gradient $|\nabla \phi|$ is not directly regularized as the latter. (In the report of Li et al., ${ }^{18}|\nabla \phi| \approx 1$ within a narrow band around the zero level set and $|\nabla \phi| \approx 0$ outside a narrow band.) $|\nabla \phi|$ in our case is bounded, i.e. $\rho_{\mathrm{s}}<|\nabla \phi|<2 \tau / \Delta x$ in any discrete application, where $\Delta x$ is the pixel size. It should be pointed out that in Li's method, $|\nabla \phi|$ is not guaranteed to be 1 unless the object and background are both 265 homogenous. Otherwise, the external force will not be zero and the regularization term cannot be zero $(|| \nabla \phi||=1)$ either. By introducing this distance regularization term, the re-initialization step is no longer required, and the level set will finally converge, given proper parameter settings for region-based or gradient-based level set functions. The convergence can then be detected using the proposed coherent propagation algorithm as demonstrated in the results section below.

$$
\begin{gathered}
E=E_{\text {internal }}+E_{\text {external }}+E_{\text {regularizing }} \\
E_{\text {regularizing }}=\int \min (|\phi-\tau|,|\phi+\tau|) d x
\end{gathered}
$$

$$
\frac{\partial \phi}{\partial t}=v_{t}+\lambda d_{r}\left(\phi, v_{t}\right)
$$




$$
d_{r}\left(\phi, v_{t}\right)= \begin{cases}\operatorname{sign}(\phi)(\tau-|\phi|) & \text { if }|\phi|>\tau \text { or }\left(|\nabla \phi|<\rho_{s}\right) \\ 0 & \text { otherwise }\end{cases}
$$

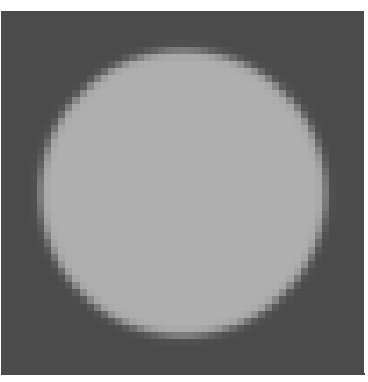

(a)

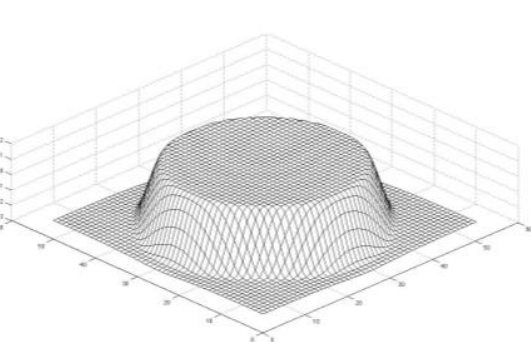

(b)

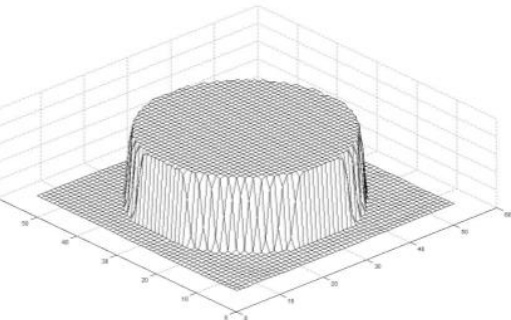

(c)

275 Figure 3. (a) Input Image, (b) Level set function after 150 iterations using Li's method. (c) Level set function after convergence using the proposed distance regularization term.

\section{Experiments and Results}

Two versions of coherent propagation algorithms, a sparse field coherently propagating level set (SF-cp) with re-initialization, and a distance-regularized coherently propagating level set (DR-cp)

on dense field, were implemented. The former was compared with the conventional sparse field algorithm (SF) and the latter was compared with the dense field level set algorithm with additional distance regularization term (DR). A region-based level set function, using Eq. 2, was used in all the tests. To explore the properties of coherently propagating level sets, three experiments using synthetic patterns were designed.

285 The first two experiments were based on a test pattern of a disc with a radius of 250 while the image size is $512 \times 512$. In the first experiment, the parameters of level set propagation were kept unchanged while random noise of different magnitudes was added (e.g. Fig. 4). In the second experiment, image noise was kept unchanged, while the weighting factor on the curvature force was gradually increased. In the third experiment, a series of star-shaped patterns, defined by the polar

290 function $r=\eta \sin (6 \theta-\pi / 2)+150$, were used. The series was created by gradually increasing $\eta$ from 10 to 100 (e.g. Fig. 5). The initial seed region for these three experiments is a circle located in the disc center with a diameter of 75 . For the SF and DR algorithms, the number of iterations was 
determined manually by a human observer. The running time of different algorithms and the number of iterations required are plotted in Figs. 6, 7, 8, together with the average distance (mean \pm standard deviation) to the ground truth of algebraic solutions created in Matlab.

In the above experiments, a damping-factor-based convergence strategy was used, $d$ was set to 0.5 , and $s_{\min }$ was set to 0.1 . For simplicity, the neighborhood size is set to infinite, i.e. a global synchronized trend is used in all test cases.

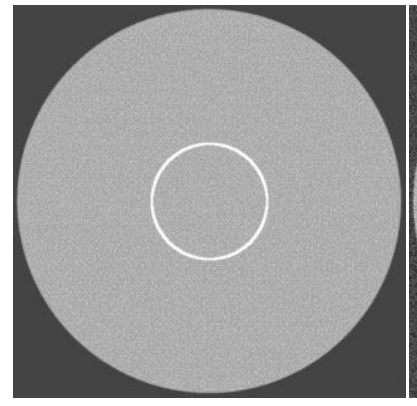

(a)

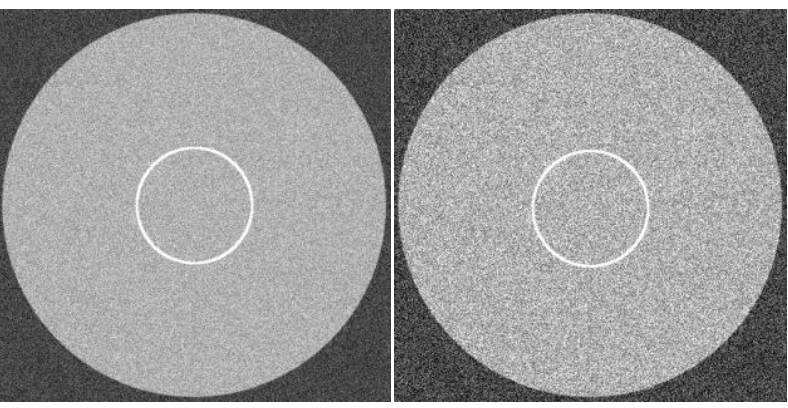

(c)

Figure 4. Synthetic patterns for experiment 1 and 2. (a) Original image without noise (object intensity: 175 background: 75). (b) Add random noise (maximum noise magnitude: 40). (c) Add random noise (maximum noise magnitude: 75). For both experiments, the threshold window width of the external speed term was set to 100 and centered at 175 . White circles are initial regions

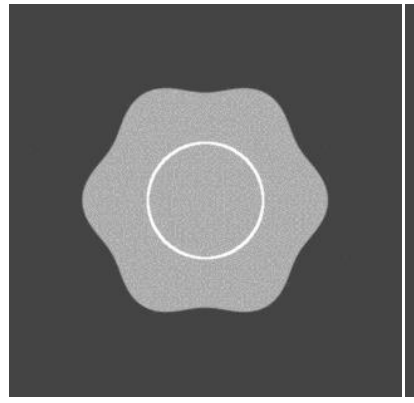

(a)

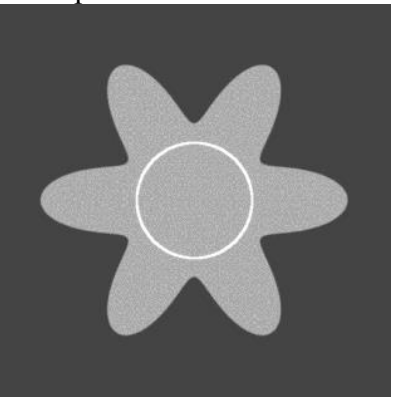

(b)

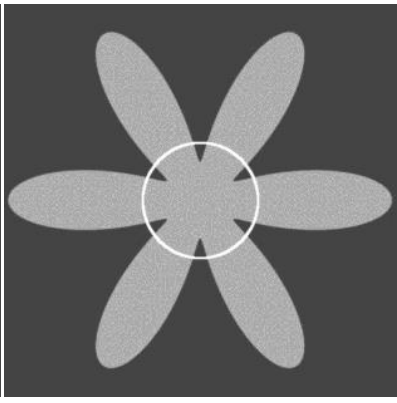

(c)

Figure 5. Synthetic pattern for Experiment 3. (a) $\eta=10$, (b) $\eta=50$, (c) $\eta=100$. White circles are initial regions

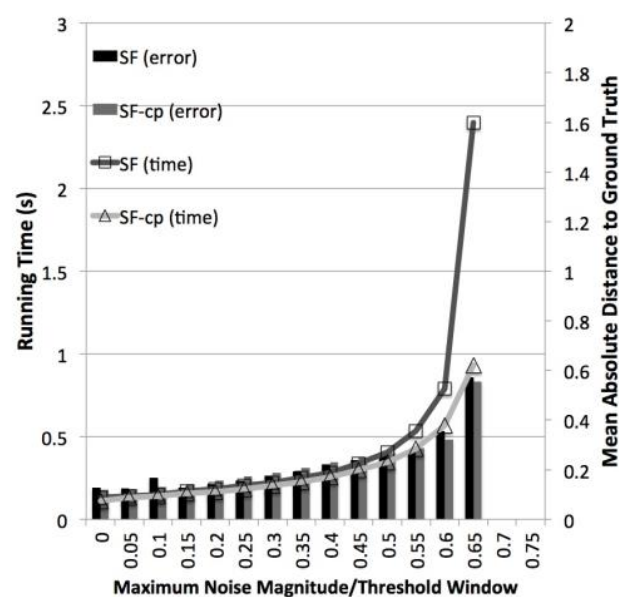

(a)

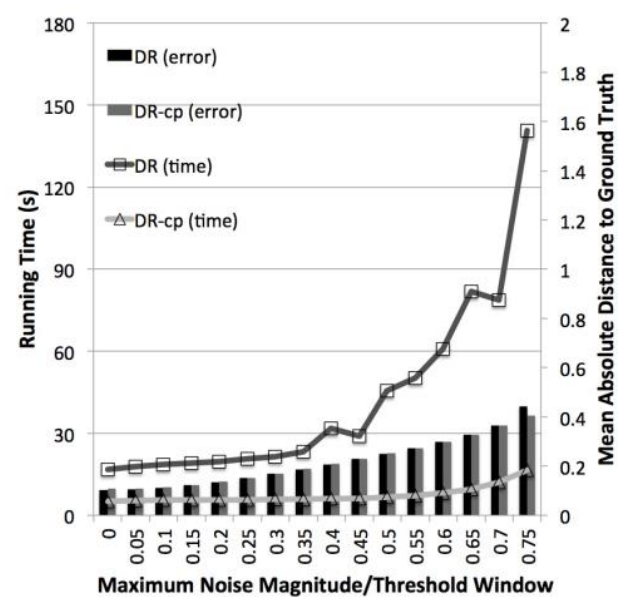

(b)

Figure 6. Results from Experiment 1. (a) comparing the running time and accuracy between SF and SF-cp algorithms. (b) comparing the running time and accuracy between DR and DR-cp algorithms. 


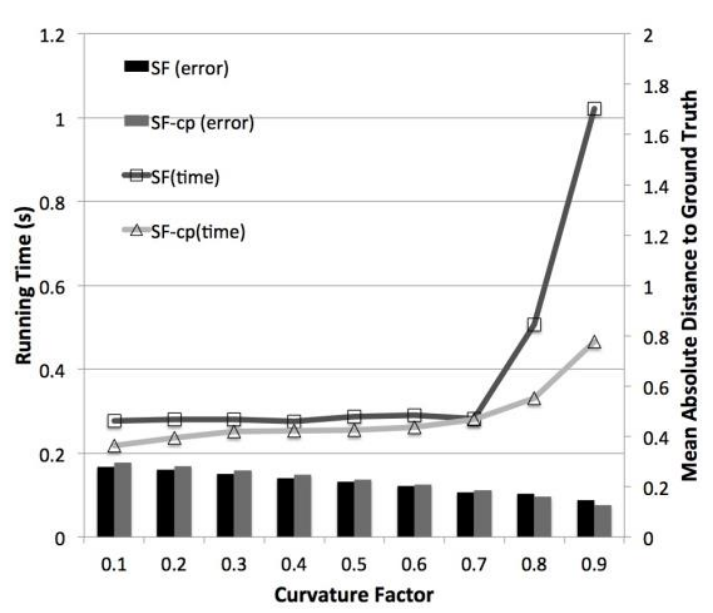

(a)

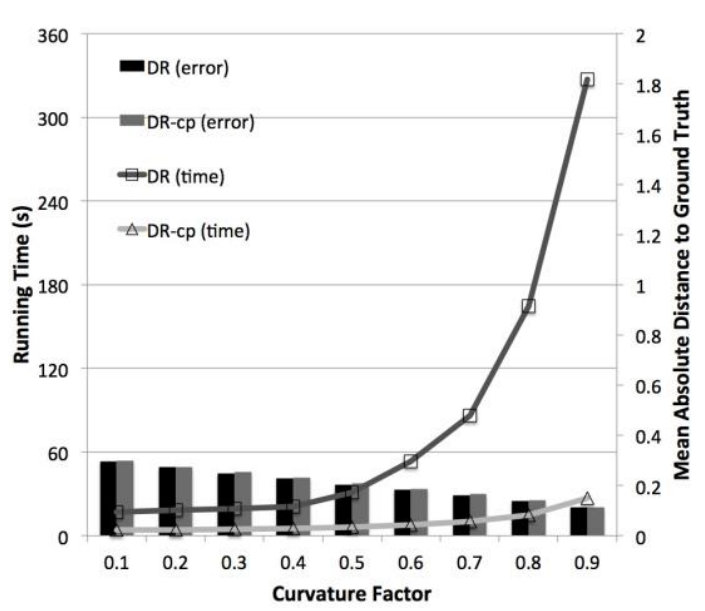

(b)

Figure 7. Results from Experiment 2. (a) comparing the running time and accuracy between SF and SF-cp algorithms. (b) comparing the running time and accuracy between DR and DR-cp algorithms.

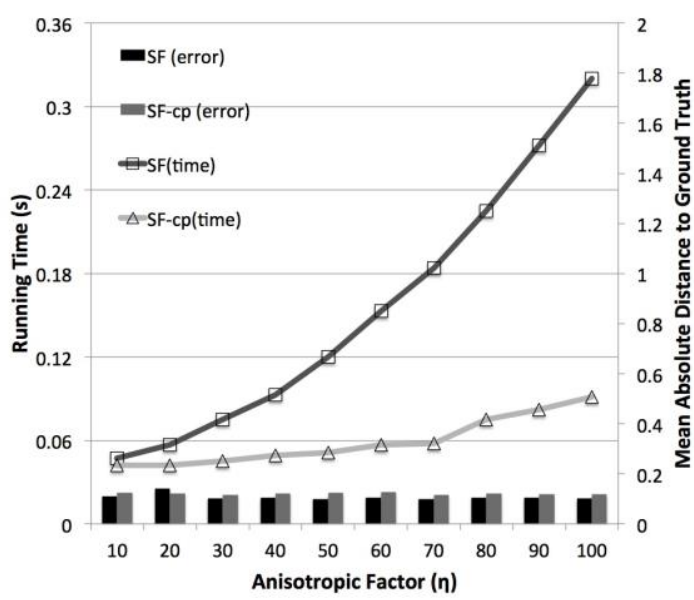

(a)

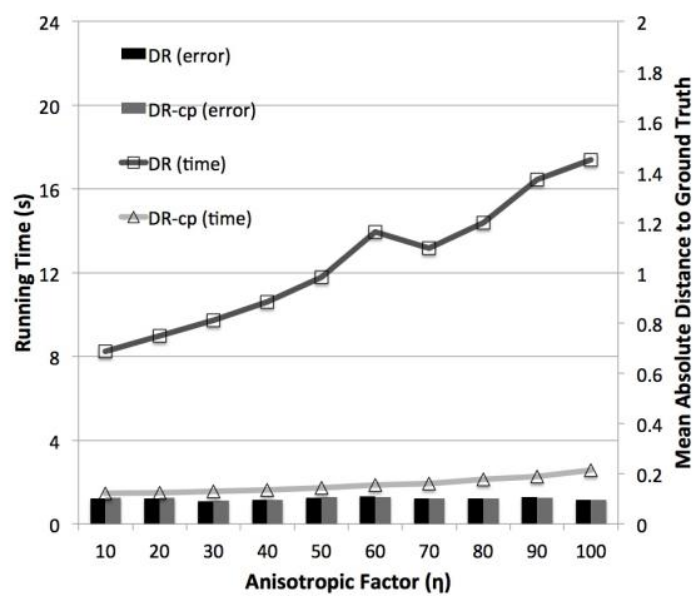

(b)

Figure 8. Results from Experiment 3. (a) comparing the running time and accuracy between SF and SF-cp algorithms. (b) comparing the running time and accuracy between DR and DR-cp algorithms.

Seven brain T1W MRI datasets, twelve abdominal CT angiography datasets and ten liver enhancement abdominal CT were used to test the performance of the SF-cp algorithm on 3D data. The same region-based level set function as used in the 2D cases was used. The curvature factor $(\alpha)$ was set to 0.5 for all cases, while the thresholds were manually determined for each case by an experienced user. In all cases, a single sphere seed region was put inside the organ where the diameter is as large as possible (cf. dark circles in Fig. 9). Side branches of aorta and organs attached to liver, where leaking may occur, are cropped away manually before running the segmentation algorithms (cf. white circles in Fig. 9). The segmentation performance and accuracy was compared with the medical segmentation software ITKSnap, ${ }^{1}$ which implements a sparse field 
level set method. Both softwares were running in a single-threaded mode on an Intel Core i7 CPU. The experiment results show that the Dice coefficient between the SF-cp algorithm and the SF algorithm (implemented in ITKSnap) was above 99\% in almost all cases when the same parameters, seed regions and crop regions were used. In the liver cases, the segmentation results were also 335 compared with manual segmentation results, which were created via slice-by-slice manual drawing. The Dice coefficient between the SF-cp algorithm and the "ground truth" was $97.1 \pm 2.8 \%$. While the user-interaction time was around 1-2 minutes for both softwares with key parameters predetermined, the level set propagation time was drastically shorter with the SF-cp algorithm. The speed gain of the new algorithm ranged between 8 and 16 times for brain segmentation, between 71 340 and 251 times for aorta segmentation and between 31 and 87 times for liver segmentation. Detailed results of the experiments are summarized in Table III. Fig. 10 compares the number of active points (updated points) in each iteration between the SF-cp and SF algorithm. Our testing software, MiaLite, is freely available online at http://www.cmiv.liu.se/output/software. The online version is optimized to run parts of the algorithm in parallel on the CPU, increasing speed by a factor of 2-3 on our quad-core processor. The average processing time using multi-threaded SF-cp algorithm was $18.0 \pm 2.7 \mathrm{~s}, 2.8 \pm 1.1 \mathrm{~s}$ and $19.5 \pm 5.3 \mathrm{~s}$, respectively, for segmenting brains, aortas and livers.

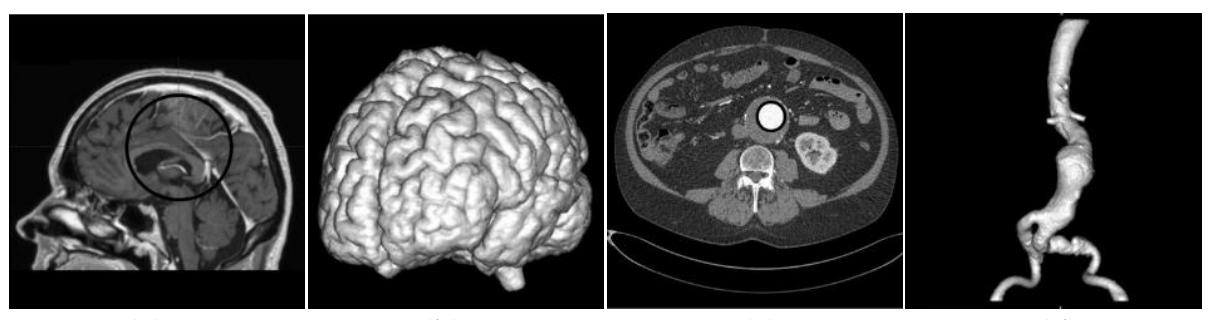

(a)

(b)

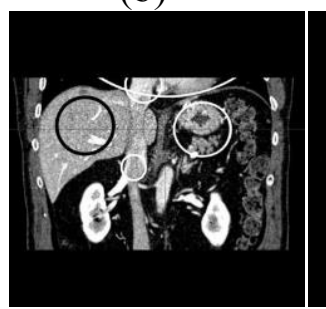

(e) (c)

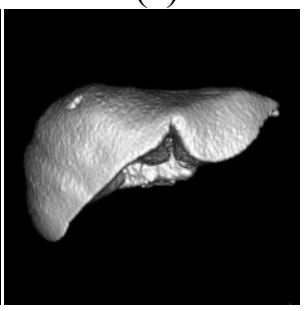

(f)

(d)

Figure 9. Segmentation examples. (a), (b), (c): original slices. (c), (d), (f): segmentation results rendered in 3D. Black circles are seed regions, White circles are cropped-away regions 
Table III Experiments on medical data. Running times and speed-up factors with SF and SF-cp algorithms

\begin{tabular}{|c|c|c|c|c|c|c|}
\hline \multirow{2}{*}{$\begin{array}{l}\text { Organ } \\
\text { (cases) }\end{array}$} & \multirow{2}{*}{ Modality } & \multirow{2}{*}{ Size } & \multicolumn{2}{|c|}{ Running time } & \multirow{2}{*}{$\begin{array}{l}\text { speed-up } \\
\text { factor } \\
\text { (times) }\end{array}$} & \multirow{2}{*}{$\begin{array}{c}\text { Dice } \\
\text { coefficient }\end{array}$} \\
\hline & & & SF-cp & SF (ITK) & & \\
\hline Brain (7) & MRI & $256^{2} \times 140 \sim 240$ & $47.8 \pm 8.0 \mathrm{~s}$ & $517 \pm 84 \mathrm{~s}$ & $11 \pm 2$ & $98.9 \pm 0.3 \%$ \\
\hline Aorta (12) & CT & $512^{2} \times 350 \sim 500$ & $5.0 \pm 1.5 \mathrm{~s}$ & $500 \pm 138 \mathrm{~s}$ & $107 \pm 31$ & $99.4 \pm 0.1 \%$ \\
\hline Liver* (10) & CT & $512^{2} \times 220 \sim 430$ & $51.2 \pm 16.5 \mathrm{~s}$ & $39.5 \pm 12.3 \mathrm{~min}$ & $49 \pm 12$ & $99.3 \pm 0.2 \%$ \\
\hline
\end{tabular}

*A multi-resolution approach was used in the coherent propagation cases, but single-resolution implementation in ITK

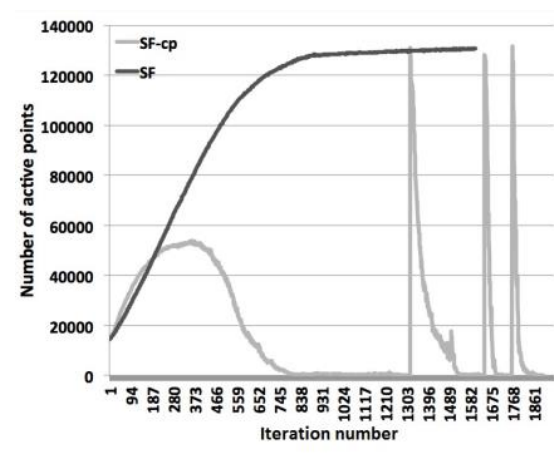

(a)

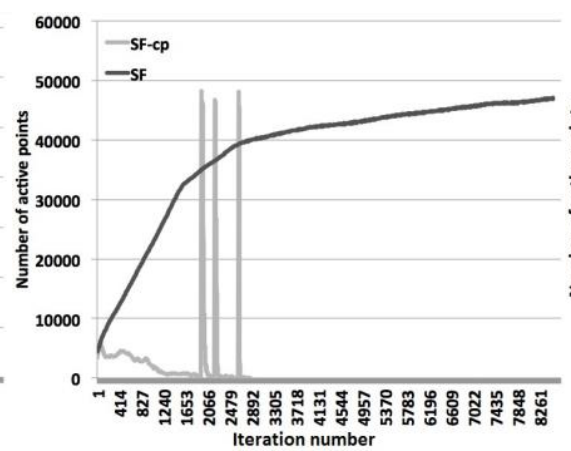

(b)

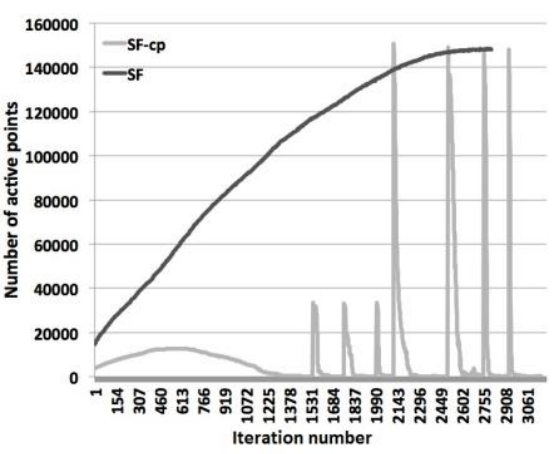

(c)

Figure 10. Comparison of the number of active points on the narrow band during the level set propagation with SF and the SF-cp algorithms in 3D cases. (a) a typical example of brain segmentation. (b) a typical example of aorta segmentation. (c) a typical example of liver segmentation. Note: the peaks in the SF-cp plots correspond to the beginning of new periods. The difference of peak height in (c) is due to different resolution.

\section{Discussion and Conclusions}

The level set method is a powerful segmentation tool, but in many applications, e.g. brain segmentation in MRI, the parameters in the speed function have to be tuned for each dataset. Letting the user wait for several minutes before seeing the results of changes is unacceptable for an interactive process. Therefore, making the level set method faster is of great interest to many image processing researchers as well as practitioners. One factor making the level set method slow is due to the difficulty to detect convergence of the level set function. The most common practice is to stop the evolution after a fixed number of iterations. A relatively high number has to be set to guarantee the convergence if the shape of the object and the traveling speed of the contour cannot be 
estimated beforehand. This will cause prolonged running time for the majority of cases. Another

375 common approach is to set a limit on the root-mean-square error (RMSE), which is usually approximated by summing the absolute travel distance of each point in one iteration. In our experience, such a parameter is hard to control. In many cases, RMSE can be high even when the contour reaches the border of the object. A fixed RMSE might cause the propagation to end prematurely or to go on indefinitely. This issue is partly due to the discretization of the external 380 force field in which the force can change sign between two neighboring pixels/voxels. When reaching the border of the object, the zero level set will constantly jump between two pixels instead of having zero velocity. Combined with the curvature force, the wiggling behavior of the contour is almost inevitable when hitting the border, unless some interpolation of the external force field is applied.

385 The proposed method solves this problem by forcing the movement of level sets to be monotonic on each pixel/voxel, i.e., the contour can only cross a pixel/voxel once within a single period. This makes it evident when the contour has crossed the border of the targeted object. The small overshoot is solved by repeating the coherent propagation in new predicted directions, so that the jumping around the border is processed in a more controlled manner. The comparison between the manually determined iteration number in conventional methods (SF and DR) and the coherent propagation methods in Experiment 1,2 and 3 proved the ability of coherently propagating level sets to stop naturally when convergence has been obtained. Note that after the first period, each subsequent period usually adds only few iterations, as the travel distance of the contour is very short once it has reached the border in the first period. The ability to detect convergence also makes it easy to implement a multi-resolution approach, as it is clear when to move from lower resolution to higher resolution. This is demonstrated in our experiments with liver segmentation in CT datasets.

The coherent propagation approach can also result in a locally synchronized gradient descent, which stops the local wiggling movement. This occurs not only because we can define a 
synchronized trend map for neighboring pixels but also because of the nature of the level set that 400 allows the contour to change topology at certain points which are propagating in a different direction. So in the sparse field application, even if the initial trend map for the initial contour is inhomogeneous, after a few iterations the trend of the active propagating part will have the same trend as the points with opposite trend being cut away from the moving contour (become "islands"). Stopping these wiggling movements can not only save a few computations on certain pixels (less 405 than $1 \%$ of the overall computations in Experiment 1 and 2) but also allow the contour to propagate in larger steps and reach the border in fewer iterations. This benefit is more prominent in noisy cases or with higher curvature weighting factor, as shown by the results from Experiment 1 and 2 (Fig. 5, 6).

Another, even more important, advantage of coherent propagation is the ability to detect local 410 convergence and thus skip unnecessary calculations while the rest of the contour can continue to grow. Although this advantage is not noticeable in cases where the contour hits the entire border almost simultaneously, e.g. in Experiment 1 and 2, it can lead to great speed-up when certain parts of the contour need to propagate longer distances than other parts. This makes it more attractive in cases where the shape to be segmented is asymmetric, as in Experiment 3. Besides, the initial seed 415 region can also affect whether the contour can hit the whole border simultaneously. For example, in Experiment 1 and 2, if the initial seed region is shifted from the exact center of the circular pattern, the performance difference will increase thanks to the local convergence detection. The benefit of reduced computation is more noticeable in $3 \mathrm{D}$ cases as shown in Fig. 10. Again the most anisotropic structure - the aorta - gains most in terms of speed-up. However, for the most sphere420 like structure - the brain - the computation reduction is still significant. Roberts et al. ${ }^{9}$ also proposed a method to detect local covergence by judging whether $\Delta t \times v_{t}$ on one point is canceled out in two consecutive iterations (requiring $\Delta t$ to be fixed). To our experience, this so-called "temporal equilibrium" is difficult to be detected due to numerical errors and not theoretically 
guaranteed to capture the local convergence, as the magnitude of jumping backwards and forwards may not necessarily be equal. In contrast, the coherent propagation approach is easier to implement and will always capture convergence once it occurs.

One drawback of coherent propagation is that the overshoot on the object border can be larger than with the conventional method. This may cause the contour to fluctuate in some areas where it will not converge, as described in Section 2.2. Here we proposed to use a damping factor to make 430 the fluctuation to stop gradually. This is somewhat similar to the shifting-cut when using the GaussNewton method to solve PDE, but here we solve the equation at multiple points. Instead of treating them independently as the conventional methods, they are grouped by the trend map, so that the shifting-cut only happens when the group has finished updating (at the end of each period). Compared with the previously proposed wiggling counting approach, ${ }^{21}$ using a damping factor is 435 easier to implement and easier to extend to non-narrow-band cases. It also reduces the total number of periods needed to stop fluctuation. In our experience, a damping factor of 0.5 gives a well balanced performance between the total number of periods (to approach $s_{\max }=0.1$ ) and the number of iterations within each period. It can, however, be tuned to gain better performance in different implementations.

440 Another interesting character of the coherent-propagating level set is that it tends to pass over local minima. In Experiment 1, it was observed that the SF-cp algorithm tends to propagate further towards the ground truth in the noisy cases, and thus have a smaller mean distance to the ground truth. When used on medical images, in our experience, coherent propagation can more easily cross a moderate stenosis in vessel segmentation (discussed in the report of Wang et al.), ${ }^{11}$ or an edema 445 region in the brain. When using conventional level sets, the threshold window (Eq. 1) has to be carefully tuned to achieve equivalent results. Dense field level sets are even more prone to global optimization: as shown in Fig. 5, the disk was correctly segmented even with aggressive noise. This makes the dense field implementations more desirable in very noisy cases, or cases with relatively 
high noise compared to the segmentation threshold window ( $\varepsilon$ in Eq. 2.), which sometimes has to be set to very narrow to avoid the leaking problem. By introducing the distance regularization term, we ensure the convergence of the level set function. This property is then used by the coherent propagation to skip most computations when they go beyond the "floor and ceiling". In one period, this can be seen as updating a narrow band implicitly; however, this "narrow band" is the naturally defined actively changing points instead of points that are near the zero level set. The distance regularization approach also eliminates the need of re-initialization as $|\nabla \phi|$ is bounded. Compared with Li's method, our method does not regularize the gradient of the level set function directly, but the ||$\nabla \phi||$ is still bounded by the resolution of the discretized level set function. With Li's solution, the floor and ceiling can be drifting upward or downward in small steps due to inevitable numeric errors at convex or concave sharp corners in the images, and therefore the advantage of 460 using coherent propagation is limited since local convergence detection cannot be achieved. Regularizing the distance directly can not only make the convergence more evident, but also simplify the overall computation. Note that the distance regularization term is similar to the data fidelity term of the $\mathrm{L}^{1}$ total variation model. ${ }^{25}$ This also suggests the possibility of using the coherent propagation for some de-noising algorithms, but this potential has not been evaluated in 465 our current study.

One limitation of the MiaLite software is the need to manually crop away some adjacent organs to achieve reasonable segmentation accuracy. It follows the same design philosophy of ITKSnap, i.e., to gain flexibility by allowing a reasonable amount of user interaction. For specific applications, we have also developed a number of automatic model-based level-set segmentation 470 methods combining coherent propagation with prior anatomical knowledge. ${ }^{26-28}$ The thresholdbased level set function is one of the simplest region-based level set functions. The simplicity makes parameter-tuning relatively easy even for clinical users. In our experience, the thresholds for vessel segmentation in CTA do not vary much from case to case. For liver segmentation and brain 
segmentation, the thresholds need to be manually tuned for each case using a similar user interface as proposed by Lefohn et al. ${ }^{19}$ However, the manual parameter tuning could also be replaced by histogram analysis or other types of parameter estimation techniques. Another common practice of image segmentation is to convert image features to a probability map; e.g., threshold-based level set is a popular choice to finish the final segmentation using the probability map. One successful example is tumor segmentation: given a marked tumor region (for instance a line across the tumor),

480 a learning-based algorithm can learn the appearance of the tumor voxels and map every voxel to a probability value from -1 to 1 , and then the level set method is used to retrieve a relatively smooth region where the majority of voxels have positive values ${ }^{29}$. Although our experiments focused on region-based level set methods with fixed threshold, the coherent propagation approach can be extended easily to use gradient-based external speed. From our preliminary experience, the 485 performance difference is about same as that found in our presented experiments. Extending this to the Chan-Vese method, however, is less straightforward, as the external force will change in each iteration and all points have to be woken up in each iteration, so that the only benefit is a slightly larger step length, as indicated by Experiment 1. To be able to skip local converged points, a simple strategy is to delay the update of the external force until the end of each period and set the maximum number of iterations within one period ( $n$ in Table II) to a relatively small number, e.g. 50. Here, a heterogeneous trend map is recommended, and it needs to be re-estimated every period. The damping factor is only applied when the majority of points (a given percentage) fall asleep within one period. Although this strategy requires more computation than the threshold-based level set, substantial speed-up can be achieved in cases where the object has a very irregular shape.

495 Judging from the published running time of various fast level set algorithms, the speed of the proposed algorithm is superior to most state-of-the-art fast level set algorithms except for Gibou's hybrid k-means level set ${ }^{11}$ and Shi's approximated level-set-based curve evolution method. ${ }^{13}$ Both these algorithms de-couple the image term from the regularization term during the level set 
evolution when solving the PDE. In other words, their approaches are similar to running a region

500 growing first without any local shape constraint and then perform a Gaussian smoothing. Therefore, the behavior of the region propagation is very different from the original level set. One of obvious drawbacks is the leaking problem, which means the propagating region will enter a nearby undesired object via a small tunnel between the objects. This is often seen in medical images, due to limited resolution. The leaking can be avoided with the conventional level set methods given a proper weighting factor on the curvature term, but is difficult with Gibou's or Shi's methods. In Shi's report, ${ }^{13}$ a lower Dice coefficient (95.6\%) was reported, even though they were segmenting only white matter from a noise-free simulated MRI dataset, while our algorithm agrees better with the conventional level set method when segmenting the whole brain including the gray matter (more challenging than white matter only due to the leaking problem) in real patient data.

510 In conclusion, we have presented a generalized coherent propagation algorithm for level set evolution. The new algorithm implements a damping factor to sequentially reduce the maximum step length of the evolution and stops the fluctuation of the moving contour. Finally, the coherent propagation approach is combined with a new distance-regularized level set, which eliminates the needs of re-initialization of the distance. Substantial improvement in processing time was observed 515 with both synthetic datasets and medical images.

\section{References}

1 P.A. Yushkevich et al., "User-guided 3D active contour segmentation of anatomical structures: significantly improved efficiency and reliability," Neuroimage 31(3), 1116-1128 (2006).

2 E. Ukwatta et al., "Three-dimensional segmentation of three-dimensional ultrasound carotid atherosclerosis using sparse field level sets," Med Phys 40(5), 052903 (2013).

3 W. Qiu, J. Yuan, E. Ukwatta, D. Tessier, and A. Fenster, "Three-dimensional prostate segmentation using level set with shape constraint based on rotational slices for 3D end-firing TRUS guided biopsy,” Med Phys 40(7), 072903 (2013). 
4 H. Tang, T. van Walsum, R. Hameeteman, R. Shahzad, L.J. van Vliet, and W.J. Niessen, "Lumen segmentation and stenosis quantification of atherosclerotic carotid arteries in CTA utilizing a centerline intensity prior," Med Phys 40(5), 051721 (2013).

5 D. Adalsteinsson and J.A. Sethian, "A Fast Level Set Method for Propagating Interfaces," J. Comput. Phys. 118, 269-277 (1994).

5306 R.T. Whitaker, "A level-set approach to $3 \mathrm{~d}$ reconstruction from range data," Int. J. Comput. Vision 29, 203-231 (1998).

7 J. Deng and H.-T. Tsui, "A fast level set method for segmentation of low contrast noisy biomedical images," Pattern Recognition Lett. 23(1), 161-169 (2002).

D.T. Vu, J.Y. Kim, S.H. Choi, and S.Y. Na, "Image segmentation using a generalized fast level set method," in Proceedings of the 9th WSEAS international conference on Signal processing, robotics and automation(2010), pp. 298-303.

9 M. Roberts, J. Packer, M.C. Sousa, and J.R. Mitchell, "A work-efficient GPU algorithm for level set segmentation," in Proceedings of the Conference on High Performance Graphics(2010), pp. 123-132.

54010 R. Goldenberg, R. Kimmel, E. Rivlin, and M. Rudzsky, "Fast geodesic active contours," IEEE Trans. Image Process. 10(10), 1467-1475 (2001).

11 F. Gibou and R. Fedkiw, "A fast hybrid k-means level set algorithm for segmentation," in Proceedings of the 4th Annual Hawaii International Conference on Statistics and Mathematics(2002), pp. 281-291.

54512 Y. Shi and W.C. Karl, "A fast level set method without solving PDEs," in Proceedings of the IEEE International Conference on Acoustics, Speech, and Signal Processing(2005), pp. 97100.

13 Yonggang Shi and W.C. Karl, "A Real-Time Algorithm for the Approximation of Level-SetBased Curve Evolution," IEEE Trans. Image Process. 17(5), 645-656 (2008). O. Bernard, D. Friboulet, P. Thevenaz, and M. Unser, "Variational B-Spline Level-Set: A Linear Filtering Approach for Fast Deformable Model Evolution,” IEEE Trans. Image Process. 18(6), 1179-1191 (2009).

15 B. Nilsson and A. Heyden, "A fast algorithm for level set-like active contours," Pattern Recognition Lett. 24(9), 1331-1337 (2003).

55516 Y.-H.R. Tsai, "Threshold dynamics for the piecewise constant Mumford-Shah functional," J. Comput. Phys. 211(1), 367-384 (2006).

17 M. Maska, P. Matula, O. Danek, and M. Kozubek, "A fast level set-like algorithm for regionbased active contours," in Advances in Visual Computing(Springer, 2010), pp. 387-396.

18 C. Li, C. Xu, C. Gui, and M.D. Fox, "Distance Regularized Level Set Evolution and Its 560 Application to Image Segmentation," IEEE Trans. Image Process. 19(12), 3243-3254 (2010).

19 A. Lefohn, J. Cates, and R. Whitaker, "Interactive, gpu-based level sets for 3D segmentation," in Proceedings of the MICCAI conference (2003), pp. 564-572.

20 F. Galluzzo, N. Speciale, and O. Bernard, "A rigorous and efficient GPU implementation of level-set sparse field algorithm," in Proceedings of the 19th IEEE International Conference

21 C. Wang, H. Frimmel, and O. Smedby, "Level-set based vessel segmentation accelerated with periodic monotonic speed function," in Proceedings of the SPIE Medical Imaging Conference (2011), p. 79621M-79621M-7.

22 S. Osher and R.P. Fedkiw, Level set methods and dynamic implicit surfaces (Springer, 2003). T.F. Chan and L.A. Vese, "Active contours without edges," IEEE Trans. Image Process. 10(2), 266-277 (2001).

24 V. Caselles, R. Kimmel, and G. Sapiro, "Geodesic active contours," Int. J. Comput. Vision 22(1), 61-79 (1997). 
25 W. Yin, D. Goldfarb, and S. Osher, "The Total Variation Regularized L1 Model for Multiscale Decomposition," Multiscale Model. Simul. 6(1), 190-211 (2007).

26 C. Wang, R. Moreno, and Ö. Smedby, "Vessel segmentation using implicit model-guided level sets," in the 3D Cardiovascular Imaging: a MICCAI segmentation challenge workshop (Nice, France, 2012).

27 C. Wang and Ö. Smedby, "Fully automatic brain segmentation using model-guided level sets and skeleton-based models," The MIDAS Journal (2013).

28 C. Wang and Ö. Smedby, "Automatic multi-organ segmentation in non-enhanced CT datasets using Hierarchical Shape Priors," in ICPR 2014 (Accepted) (2014).

29 D. Smeets, D. Loeckx, B. Stijnen, B. De Dobbelaer, D. Vandermeulen, and P. Suetens, "Semiautomatic level set segmentation of liver tumors combining a spiral-scanning technique with supervised fuzzy pixel classification," Medical Image Analysis 14(1), 13-20 (2010). 